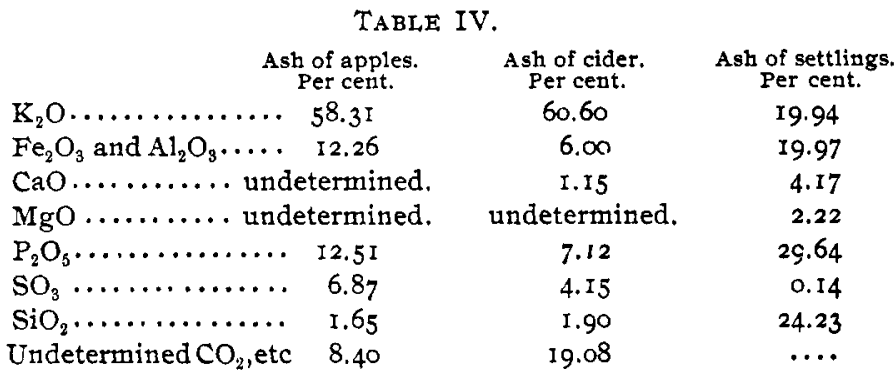

The high percentage of silica and phosphoric acid in the ash of the settlings is noteworthy; no carbonates were present.

In concluding, the writer expresses acknowledgment to Messrs. C. P. Beistle and A. N. Diehl, former assistants at this experiment station, for help in much of the analytical work of the foregoing experiments, and also to Dr. William Frear for his encouragement and many kindly suggestions, and under whose initiative the work was first begun. The writer owes an especial debt of gratitude to his former teacher, Professor Alfred Koch, of Göttingen University, for much valuable advice and information upon this subject of fermentation.

[CONTRIBUTION FROM THE CHEMICAL LABORATORY OF THE UNIVHRSITY of Texas, Austin, Texas.]

\title{
A CONTRIBUTION TO THE CHEMISTRY OF FATIGUE.
}

BY HENRY WINSTON HARPER AND MARGARET HOLLIDAY.

Received September 22, 1902.

INTRODUCTION.

SoME years since, while engaged in the study of the phenomena of fatigue and breathlessness, the senior author of this contribution was struck with an apparent resemblance to the phenomena encountered when caffeine is administered in toxic doses. It seemed clear that the phenomena of fatigue were largely due to the action of a chemical substance present in toxic quantity in the bloodstream, and that this substance had its origin in the muscles, i. e., that the metabolism within the muscles undergoing excessive exercise was accompanied with a production of incompletely 
oxidized chemical complexes, that these complexes possessed toxic qualities, and, transported by the blood-stream throughout the body, gave rise to the phenomena grouped under fatigue. Fatigue then arises from autointoxication, but the toxic substances are not the simple end-products, urea, carbon dioxide, etc., but are far more complex bodies, among which may possibly be found one or more of the alkyl derivatives of xanthine. A preliminary investigation pointed to the presence of methyl derivatives of xanthine, and encouraged further prosecution of the inquiry; but multitudinous duties stopped the work before fruitful results were obtained. The research was not again entered upon until during the session of I9OI-I902, when the present investigation was undertaken in conjunction with Miss Margaret Holliday.

It should be distinctly understood that the results here presented are not offered as a solution of the problem of fatigue, but the data thus far accumulated are of sufficient interest to justify publication, and they represent at least a modest contribution to the chemistry of the subject. The main problem will be further investigated.

Lombard" states that: "The working cell liberates energy at the expense of its store of nutriment and oxygen, and through oxidation processes forms waste-products which are poisonous to its protoplasm. The fatigue which results from functional activity has, therefore, a twofold cause, the decrease in energy-holding compounds available for work and the accumulation of poisonous waste-matters." A similar statement is made by other physiologists; but it will be seen that such statements are only broad generalizations, and when specific information concerning the nature of the waste-products and the chemistry of their formation and action is sought, it then becomes apparent that a very large field for chemical research needs to be more deeply explored. It is equally clear that this field of investigation is full of difficulties, and, therefore, presents opportunities for numerous investigators. Like all biochemical problems, it includes a large amount of virgin soil.

The main object of this particular research was to shed some light upon the chemical nature of the metabolites which gain access to the blood-current during excessive muscular exertion.

1 Lombard: "An American Text-Book of Physiology." Igor, Vol. II, p. 70. 
It was not expected that anything approaching a solution of the problem would be speedily gained, but a hope of progress was entertained, and a definite plan of work for the future has been evolved. The question of the production of the methyl derivatives of xanthine within the muscles at the time of excessive muscular exertion and their discharge into the blood-stream was especially investigated, and every precaution was taken to exclude the access of these bodies through extraneous sources. The results are presented upon their own merits. A fuller discussion will accompany a later investigation.

ANAIYTICAL METHODS USED.

A great deal of time was devoted to the calibration of apparatus, and to the trial of the numerous analytical methods used in biochemical work with a view to ascertain the limits of accuracy and suitability for this particular inquiry. The usual numerous disappointments were encountered; and some of the methods finally adopted are far from being wholly satisfactory. Some of the tabulated results well illustrate this statement of fact. In some instances results obtained by two or more entirely different methods are recorded.

The methods of analysis adopted by the Association of Official Agricultural Chemists ${ }^{1}$ were used for the analysis of food-stuffs. Total nitrogen of the urine and of the feces, the nitrogen in the phosphotungstic acid precipitate, in the xanthine silver precipitate, theobromin silver precipitate, and purine copper precipitate, was determined by the Kjeldahl-Gunning method; the nitrogen as urea was determined by the method of A. Braunstein, ${ }^{2}$ and also by the well-known sodium hypobromite method, and the filtrate from the phosphotungstic acid precipitate was similarly treated; nitrogen as ammonia was determined by the method of $\mathrm{O}$. Folin; the nitrogen as uric acid by the method of $\mathrm{E}$. H. Bartley; the phosphates were determined by titration with a standardized solution of a uranium salt; sulphates were determined gravimetrically as barium sulphate, following the method of $O$. Folin; the

\footnotetext{
istry, "Methods of Analysis."

2 A. Braunstein: The Analyst, 26, No. 304, p. 192.

3 Otto Folin: Ibid., 27, No. 310, p. 20.

4 E. H. Bartley: This Journal, 19,649.

5 Otto Folin: Am. Journal of Physiology, 7, ${ }_{52}$.
}

1 Bulletin No. 46, revised edition. U. S. Department of Agriculture, Division of Chem- 
chlorine was determined by Volhard's method. In the preliminary experiments with the phosphotungstic acid solution, it was found that the separation of caffeine, from mixed solutions of urea, uric acid, hippuric acid and caffeine, was more easily and completely effected when hydrochloric acid was used in place of sulphuric acid; accordingly, the phosphotungstic acid precipitate in which the nitrogen was quantitatively determined was obtained by this method. The urine (acidified with hydrochloric acid and the precipitant were both brought to the boiling-point and thoroughly mixed; and the filtration and washing of the precipitate was carried out with a hot-water funnel. The methyl derivatives of xanthine were sought by two methods: (I) that of Martin Krüger $;^{1}(2)$ that of $\mathrm{H}$. W. Harper: a measured quantity of urine is evaporated to dryness on a water-bath and the residue is extracted with successive portions of hot purified chloroform; this solution is treated with animal charcoal, filtered hot and the dissolved salts obtained by fractional crystallization. In one of the precipitates obtained by the method of Krüger the nitrogen was determined by the absolute method. Particular pains were taken to obtain and use nitrogen-free reagents, and the KjeldahlGunning determinations were carried out, as usual, with the parallel blank. The determinations were mostly made in duplicate and triplicate. The specific gravity was taken with a pycnometer. The calculations are on the basis of $\mathrm{O}=\mathrm{I} 6$ (International Table of Atomic Weights, I902).

\section{EXPERIMENTAL PART.}

Mr. M. B. W., a senior student in the University of Texas, was selected for the experiment. He was twenty years of age, five feet and eleven inches high, weighing $\mathrm{I} 34.5$ pounds, and of exemplary habits. He had never used coffee, tea, tobacco, or alcoholic liquors, nor had he tasted chocolate in six months. Throughout the period of the experiment it was not possible for any of the methyl derivatives of xanthine to gain access to his system from without.

The experiment began at 8 A.M., Sunday, May I Ith, and ended at 8 A. M., Tuesday, May zoth, continuing nine days.

The diet was planned to keep the subject in nitrogen equilib-

1 Martin Krïger: Ber. d. chem. Ges., 32, 2825. 
rium. It contained whole milk, butter, bread, eggs, Malta-vita, sugar, lemons, and now and then a banana. An accurate account was kept of the weight of the foodstuffs consumed, and each day samples of the milk and bread were taken and the nitrogen of the same determined. The nitrogen in the Malta-vita and that in the butter was likewise determined, while that in the eggs, lemons and bananas was calculated from values given in Bulletin No. 28 (revised edition ) - "The Chemical Composition of American Food Materials."'1

The experiment was divided into three periods of three days each-a period of rest, a period of excessive exercise, a second period of rest. Throughout the periods of rest the subject confined himself to the performance of his usual routine college duties. During the middle period, in addition to his usual routine, he was required each day for three days to indulge in a hurdle race until he fell exhausted.

The urine was collected in clean tared bottles, retained in a refrigerator until the output of twenty-four hours was voided, then weighed, thoroughly mixed and analyzed.

The feces corresponding to the food eaten during each of the three periods were separated in the usual manner by means of willow charcoal. Four capsules were taken with the supper of May Ioth; four capsules with the breakfast of May I4th; four capsules with the supper of May I6th; and four capsules with the breakfast of May 2oth. The feces between the first and second charcoal represented the first period; that including the second and third charcoal marked the second period; and that between the third and fourth charcoal marked the third period. The feces were weighed, dried at $60^{\circ} \mathrm{C}$., again weighed and analyzed.

Because of a misunderstanding, the pulse and temperature were not recorded during the first two days of the experiment. The observations subsequently made are recorded below.

The letters $A, B, C, D, E, F, G, H, I$ represent successive periods of twenty-four hours each; A, B, C represent the first period of rest, D, E, F, the middle period (period of overexertion), G, H, I, the last period (second period of rest).

The following tables include the more important results of the experiment.

1 "The Chemical Composition of American Food Materials," Bulletin No. 28, revised edition, U. S. Department of Agriculture. Office of Experiment Stations. 
TABLE I.-PUISE AND TEMPERATURE DURING THE EXPERTMENT.

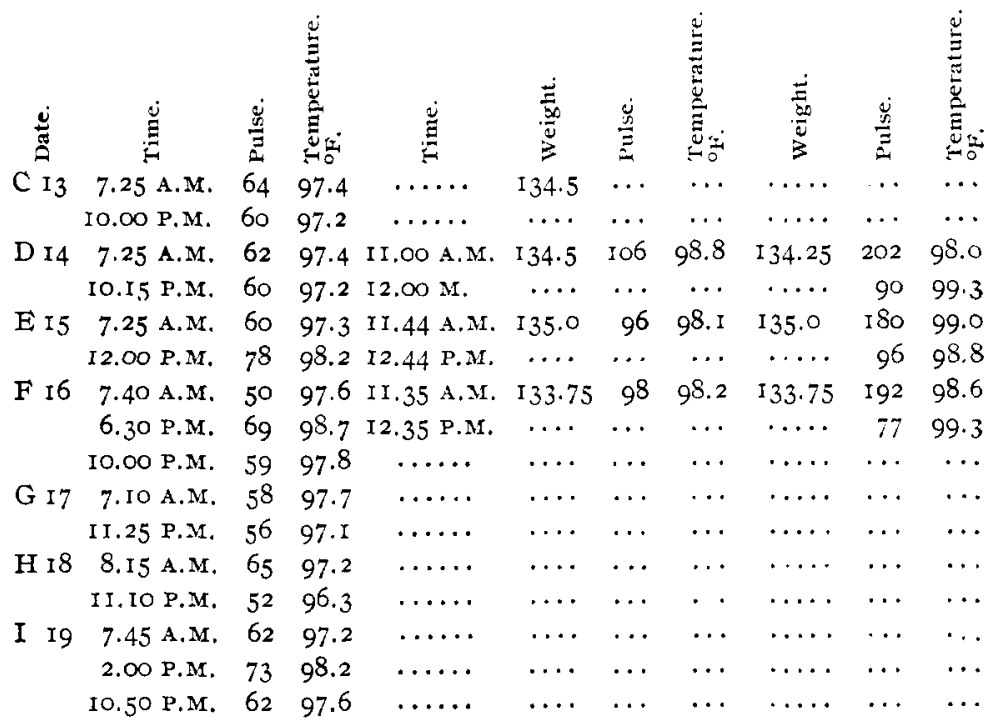

Table $I$ is self-explanatory. The influence of the excessive exercise upon the temperature is clearly shown.

TABIE II. - WEIGH AND NITROGEN CONTENT OF FOODS.

\begin{tabular}{|c|c|c|c|c|c|c|c|c|c|}
\hline \multirow{4}{*}{ 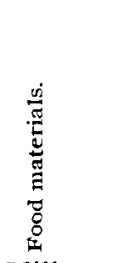 } & \multicolumn{3}{|c|}{ May il, A. } & \multicolumn{3}{|c|}{ May $\{2, \mathrm{~B}$} & \multicolumn{3}{|c|}{ May $13, \mathrm{C}$. } \\
\hline & 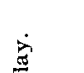 & $\stackrel{\text { 导 }}{\Xi}$ & $\stackrel{g}{g}$ & $\dot{\vec{z}}$ & $\stackrel{g}{g}$ & $\stackrel{b}{g}$ & $\dot{\mathrm{j}}$ & $\stackrel{d}{g}$ & $\stackrel{\varrho}{g}$ \\
\hline & 5 & 5 & 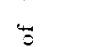 & & $\overline{8}$ & $\vec{c}$ & $\bar{\Xi}$ & $\bar{c}$ & 5 \\
\hline & 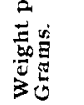 & 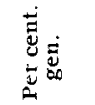 & 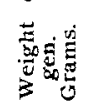 & 壳 & 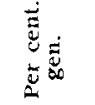 & 总嗙总 & 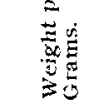 & 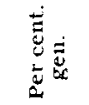 & 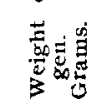 \\
\hline Milk..... J & 840.3 & $0.43^{2}$ & 7.9500 & I 864.4 & 0.432 & 8.0542 & 1633.9 & $0.5^{65}$ & 9.2315 \\
\hline Eggs .... & 344.4 & $2 . I I 2$ & 7.2737 & 267.4 & 2.112 & 5.6474 & 270.9 & $2, I I 2$ & 5.7214 \\
\hline Bread .... & 96.5 & 1.2936 & I. 2484 & 50.0 & I. 2936 & 0.6468 & 59.7 & I. 3754 & $0.82 \mathrm{II}$ \\
\hline Bread .... & $\ldots$ & $\ldots$ & $\ldots$ & 60.1 & I. 3754 & 0.8466 & 42.5 & 1.6917 & 0.7179 \\
\hline Butter ... & 17.5 & $0.35^{\circ}$ & 0.0612 & 15.5 & 0.350 & 0.0542 & 20.0 & 0.350 & 0.0700 \\
\hline Malta-vita & 51.0 & 2.918 & I. 4881 & 16.0 & 2.918 & 0.4668 & I6.3 & 2.918 & 0.4756 \\
\hline Banana .. & $\ldots$ & .. & $\ldots$ & $\ldots$ & $\ldots$ & $\ldots$ & 202.0 & 0.208 & 0.4200 \\
\hline Sugar.... & I 3.8 & $\ldots$ & $\ldots$ & 15.0 & $\cdots$ & $\ldots$ & 6.5 & $\ldots$ & $\ldots$ \\
\hline Total & & $\ldots$ & & & & .7160 & & & .4575 \\
\hline
\end{tabular}



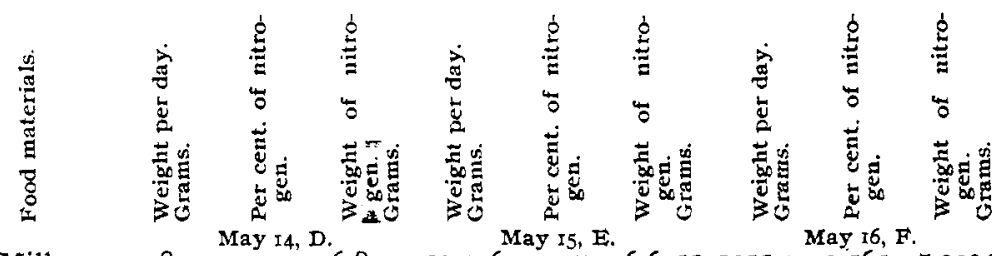

$\begin{array}{lllllllllll}\text { Milk..... I28I.I } & 0.535 & 6.8552 & 1207.6 & 0.547 & 6.6055 & \text { I } 255.3 & 0.565 & 7.0924\end{array}$

$\begin{array}{llllllllll}\text { Eggs } \ldots . . & 231.8 & 2.112 & 4.8956 & 280.9 & 2.112 & 5.9326 & 309.6 & 2.112 & 6.5387\end{array}$

$\begin{array}{llllllllll}\text { Bread .... I } 48.0 & \text { I.6917 } & 2.5037 & \text { I } 65.0 & \text { I.688 } & 2.7852 & \text { I } 51.0 & \text { I.52 } & 2.2952\end{array}$

$\begin{array}{lllllllllll}\text { Butter } \ldots & 23.5 & 0.350 & 0.0822 & 54.3 & 0.350 & 0.1900 & 33.1 & 0.350 & 0.115^{8}\end{array}$

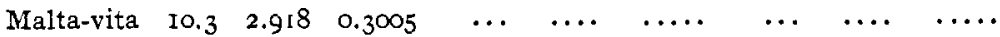

$\begin{array}{llllllllll}\text { Lemons.. } & 48.0 & 0.160 & 0.0768 & 69.0 & 0.160 & 0.1104 & 68.0 & 0.160 & 0.1088\end{array}$

$\begin{array}{lllllllllll}\text { Sugar } \ldots . & 24.5 & \ldots & \ldots & \ldots & 36.6 & \ldots & \ldots & 30.0 & \ldots & \ldots\end{array}$

Total $\mathrm{N}$ per day $\ldots . \mathrm{I} 4.7140$

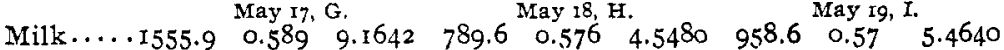

$\overline{5.6237}$

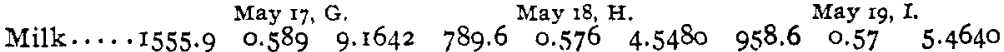

$\begin{array}{llllllllll}\text { Eggs } \ldots . . & 317.9 & 2.112 & 6.7140 & 310.9 & 2.112 & 6.5662 & 304.9 & 2.112 & 6.4394\end{array}$

$\begin{array}{llllllllll}\text { Bread .... } 54.0 & \text { T.52 } & 0.8208 & 72.0 & \text { 1.538 } & \text { I.1073 } & 52.0 & \text { I.213 } & 0.6307\end{array}$

$\begin{array}{llllllllll}\text { Bread .... } & \text { I67.0 } & 1.538 & 2.5684 & \text { I } 79.0 & \text { 1.2I3 } & 2.17 \text { I } 2 & 179.0 & \text { I.I33 } & 2.0280\end{array}$

$\begin{array}{llllllllll}\text { Butter … } & 41.6 & 0.350 & 0.145^{6} & 102.9 & 0.350 & 0.3601 & 71.9 & 0.350 & 0.2516\end{array}$

$\begin{array}{llllllllllll}\text { Malta.vita } & \ldots & \ldots & \ldots & \ldots & \text { I } 9.3 & 2.918 & 0.563 \mathrm{I} & \ldots & \ldots . & \ldots\end{array}$

$\begin{array}{llllllllll}\text { Lemons .. } & 40.0 & 0.16 & 0.0640 & 46.0 & 0.16 & 0.0736 & 49.0 & 0.16 & 0.0784\end{array}$

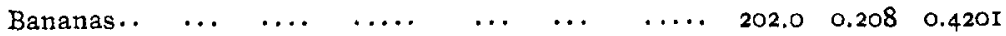

$\begin{array}{lllllllllll}\text { Sugar } \ldots & 32.0 & \ldots & \ldots & \ldots & 55.0 & \ldots & \ldots & 57.0 & \ldots & \ldots\end{array}$

$\begin{array}{llll}\text { Total N per day } \ldots & 19.4770 & 15.3895 & 15.3122\end{array}$

Table III.-Weight and Nitrogen ConTent of URINe Excreted.

Date, Weight of

May, urine.
I902. Grams.

II A $\quad{ }_{7} 84.3 \quad 24.0^{\circ} \mathrm{C}$.

I2 B II $47.3 \quad \frac{24.8^{\circ} \mathrm{C} .}{24.8^{\circ} \mathrm{C} .} \mathrm{I.0316}$

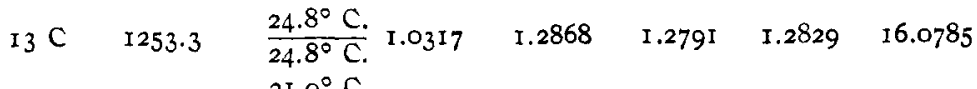

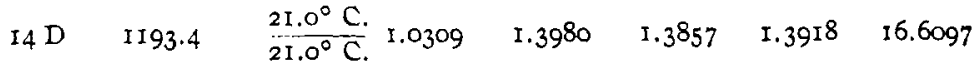

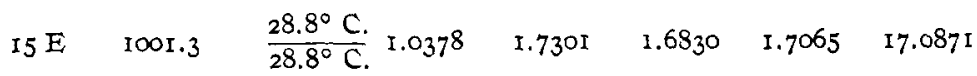

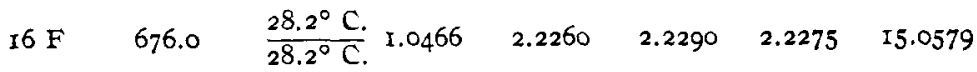

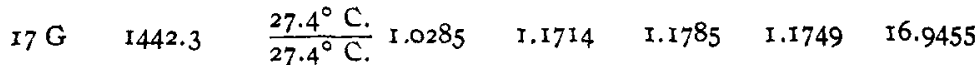

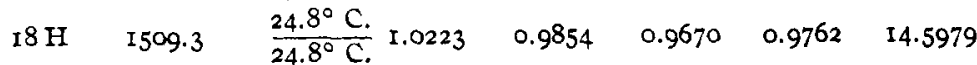

19 I $1294.0 \quad \frac{28.0^{\circ} \mathrm{C} .}{28.0^{\circ} \mathrm{C} .} 1.0304 \quad 1.0931 \quad 1.0650 \quad 1.0790 \quad 13.9622$ 


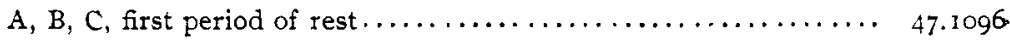

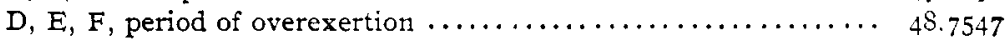

G, H, I, second period of rest...................... 45.5056

NOTE.-Each date represents the urine collected in twenty-four hours, beginning with an empty bladder at $8.00 \mathrm{A.M}$, on the dates mentioned and including all that was passed from that time to and including that in the bladder at 8.00 A.M. the succeeding day. The analytical work beyan almost immediately after the last portion of each day's output was voided.

TABLE IV.-WEIGHT AND NITROGEN CONTENT OF DRIFD FeCes.

$A, B, C$ period.

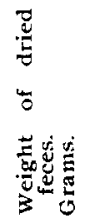

4 r.o

26.0

13.0

11.0

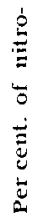

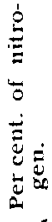

3. 169

3.434

3.0526

$3.57 \mathrm{I}$

$$
\stackrel{\dot{g}}{g}
$$

5

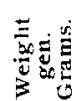

I. 2992

0.8928

0.3968

0.3928
$D, E, F$ period.

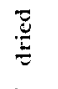

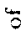

客过

30

6.0

12.0

18.0

...

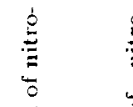

5

E

2.976

3.379

3.42

... \begin{tabular}{l}
. \\
3 \\
3 \\
0.4785 \\
0.4054 \\
0.6156 \\
$\ldots . . .5$ \\
\hline
\end{tabular}

I. 1995

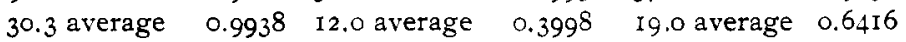

$\mathrm{G}, \mathrm{H}, \mathrm{I}$ period.

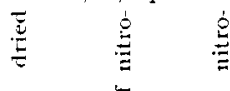

5

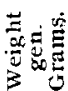

0.5110

I1.O $\quad 3.625 \quad 0.3987$

$32.0 \quad 3.173 \quad 1.0153$

‥ $\ldots ., \ldots$

\section{0}

1.9250

NoTE. - The total nitrogen of each period, divided by three, furnishes the value used in obtaining the daily nitrogen balance (see Table V).

TABIE V.-INCome and OUtgo of Nitrogen.

\begin{tabular}{|c|c|c|c|c|c|}
\hline \multirow[b]{2}{*}{$\begin{array}{c}\text { Date, } \\
\text { May, Ig02. }\end{array}$} & \multicolumn{5}{|c|}{ Nitroge11. } \\
\hline & $\begin{array}{l}\text { In food. } \\
\text { Grams. }\end{array}$ & $\begin{array}{l}\text { In feces. } \\
\text { Gram. }\end{array}$ & $\begin{array}{l}\text { In food } \\
\text { assimilated. } \\
\text { Grams. }\end{array}$ & $\begin{array}{l}\text { In urine. } \\
\text { Grams. }\end{array}$ & $\underset{\text { Gain }}{\text { Or loss }}$ \\
\hline I I $\mathrm{A}$ & I 8.02 I 4 & 0.9938 & $\mathrm{I} 7.0276$ & I 5.5965 & +1.43 I \\
\hline I2 $\mathrm{B}$ & 15.7160 & 0.9938 & I 4.7222 & I 5.4346 & -0.7 I 24 \\
\hline $\mathrm{I}_{3} \mathrm{C}$ & 17.4575 & $0.993^{8}$ & 16.4637 & I 6.0785 & -0.3852 \\
\hline $14 \mathrm{D}$ & 14.7140 & 0.3998 & 14.3142 & I 6.6097 & -2.2955 \\
\hline I5 $\mathrm{E}$ & 15.6237 & 0.3998 & I 5.2239 & $\mathrm{I} 7.087 \mathrm{I}$ & -1.8632 \\
\hline I6 F & I6.1509 & 0.3998 & $\mathrm{I} 5.75 \mathrm{I} \mathrm{I}$ & 15.0579 & $\div 0.6932$ \\
\hline $17 \mathrm{G}$ & $\mathrm{I} 9.4770$ & 0.6416 & 18.8354 & I 6.9455 & $+\mathrm{r} .8899$ \\
\hline $18 \mathrm{H}$ & I 5.3895 & 0.6416 & 14.7479 & I 4.5979 & +0.1500 \\
\hline Ig I & I 5.3122 & 0.6416 & I4. 6706 & I 3.9622 & +0.7084 \\
\hline \multicolumn{3}{|c|}{$A, B, C$, first period of rest $\ldots}$. & 48.2135 & $47 \cdot \log 6$ & +1.1039 \\
\hline \multicolumn{3}{|c|}{$\mathrm{D}, \mathrm{E}, \mathrm{F}$, period of overexertion. } & 45.2892 & 48.7547 & -3.4655 \\
\hline \multicolumn{3}{|c|}{$\mathrm{G}, \mathrm{H}, \mathrm{I}$, second period of rest... } & 48.2539 & 45.5056 & +2.7483 \\
\hline
\end{tabular}

Total gain during the experiment (nine days) .... $\quad-0.3867$ 
TABLE VI.--Nitrogen as AMMONia, AS URIC ACID, IN XaNTHINE SILver Pracipitate, in Phosphotungstic acid Precipitate, in Filtrate FROM THE Phosphotungstic acid PRecipitaty TREATED WITH SODIUM HYPOBROMITE, AS UREA (a) NaOBr Method, (b) BRaunstein's Method.

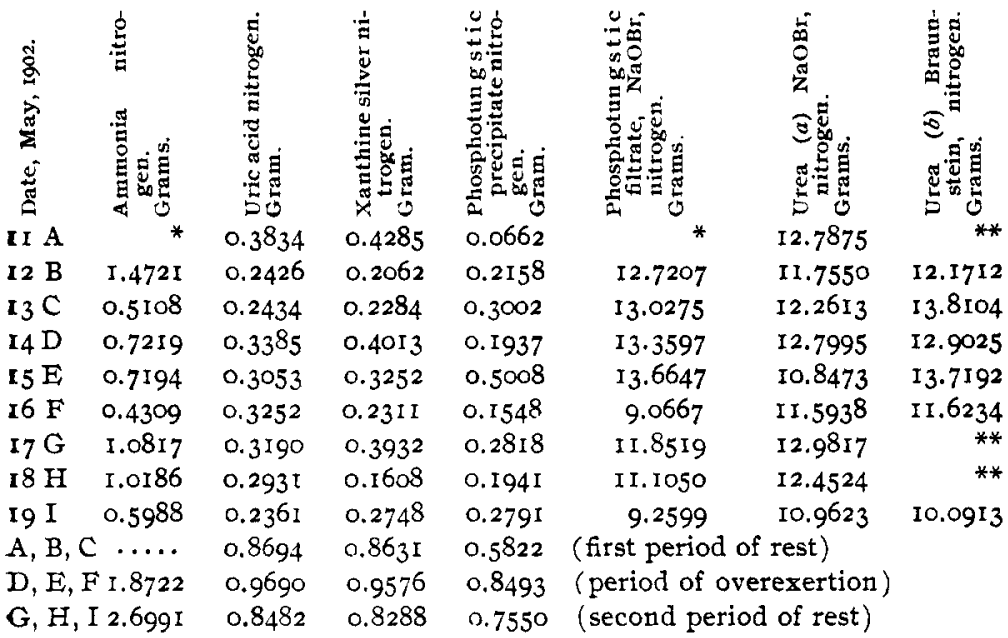

NoTE. - ${ }^{*}$ Lost; **results entirely too large to be trustworthy-discovered too late to make another trial by this method.

Until analytical methods exhibiting greater accuracy than now at our command are developed all attempts to produce a balance sheet of the nitrogenous constituents of the urine will prove unsatisfactory.

TABLE VII.-ThE $\mathrm{P}_{2} \mathrm{O}_{5}, \mathrm{SO}_{3}$, AND $\mathrm{Cl}$ CONTENT OF THE URINE.

\begin{tabular}{|c|c|c|c|c|c|c|c|}
\hline $\begin{array}{l}\text { May, } \\
\text { Igoz. }\end{array}$ & $\begin{array}{l}\text { Volume, } \\
\text { cc. at } 25^{\circ} \mathrm{C} \text {. }\end{array}$ & $\begin{array}{c}\mathrm{P}_{2} \mathrm{O}_{5} . \\
\text { Per cent. }\end{array}$ & $\begin{array}{c}\mathrm{P}_{2} \mathrm{O}_{5} . \\
\text { Grams. }\end{array}$ & $\begin{array}{c}\mathrm{SO}_{3} . \\
\text { Per cent. }\end{array}$ & $\begin{array}{c}\mathrm{SO}_{3} . \\
\text { Grams. }\end{array}$ & $\begin{array}{c}\mathrm{Cl}_{2 .} \\
\text { Per cent. }\end{array}$ & $\begin{array}{c}\mathrm{Cl}_{2 .} . \\
\text { Grams. }\end{array}$ \\
\hline II A & 1744.0 & 0.179 & 3.1247 & o. 166 & 2.9107 & 0.313 & 5.4714 \\
\hline $12 \mathrm{~B}$ & I I 15.3 & 0.263 & 2.9340 & 0.237 & $2.652 \mathrm{I}$ & 0.237 & 2.6489 \\
\hline $13 \mathrm{C}$ & I 218.3 & 0.271 & 3.3030 & 0.233 & 2.8410 & 0.260 & 3.1742 \\
\hline $14 \mathrm{D}$ & 1161.0 & 0.266 & 3.0929 & 0.250 & 2.9083 & 0.157 & $1.83 \mathrm{I} 4$ \\
\hline $15 \mathrm{E}$ & 967.5 & 0.324 & 3.1366 & 0.308 & 2.9857 & 0.228 & $2.212 \mathrm{I}$ \\
\hline $16 \mathrm{~F}$ & 647.7 & 0.404 & 2.6218 & 0.410 & 2.6555 & $0.22 \mathrm{I}$ & 1.4359 \\
\hline $17 \mathrm{G}$ & 1406.3 & 0.218 & 3.0657 & o. 197 & 2.7802 & 0.276 & 3.8885 \\
\hline $18 \mathrm{H}$ & 1481.5 & O. I 88 & 2.7948 & 0.174 & 2.5837 & 0.262 & 3.8864 \\
\hline $19 I$ & 1259.4 & 0.232 & 2.9250 & 0.197 & 2.4898 & 0.402 & 5.0671 \\
\hline$A, B, C$ & $\ldots$ & $\ldots$ & $9 \cdot 3617$ & $\ldots$ & $8.403^{8}$ & $\ldots$ & I1.2945 \\
\hline$D, E, F$ & $\ldots$ & $\ldots$ & 8.8513 & $\ldots$ & 8.5495 & $\cdots$ & $5.47^{8}$ \\
\hline $\mathrm{G}, \mathrm{H}, \mathrm{I}$ & $\ldots \ldots$ & $\ldots$ & 8.7855 & $\cdots$ & 7.8537 & $\cdots$ & I 2.8420 \\
\hline
\end{tabular}




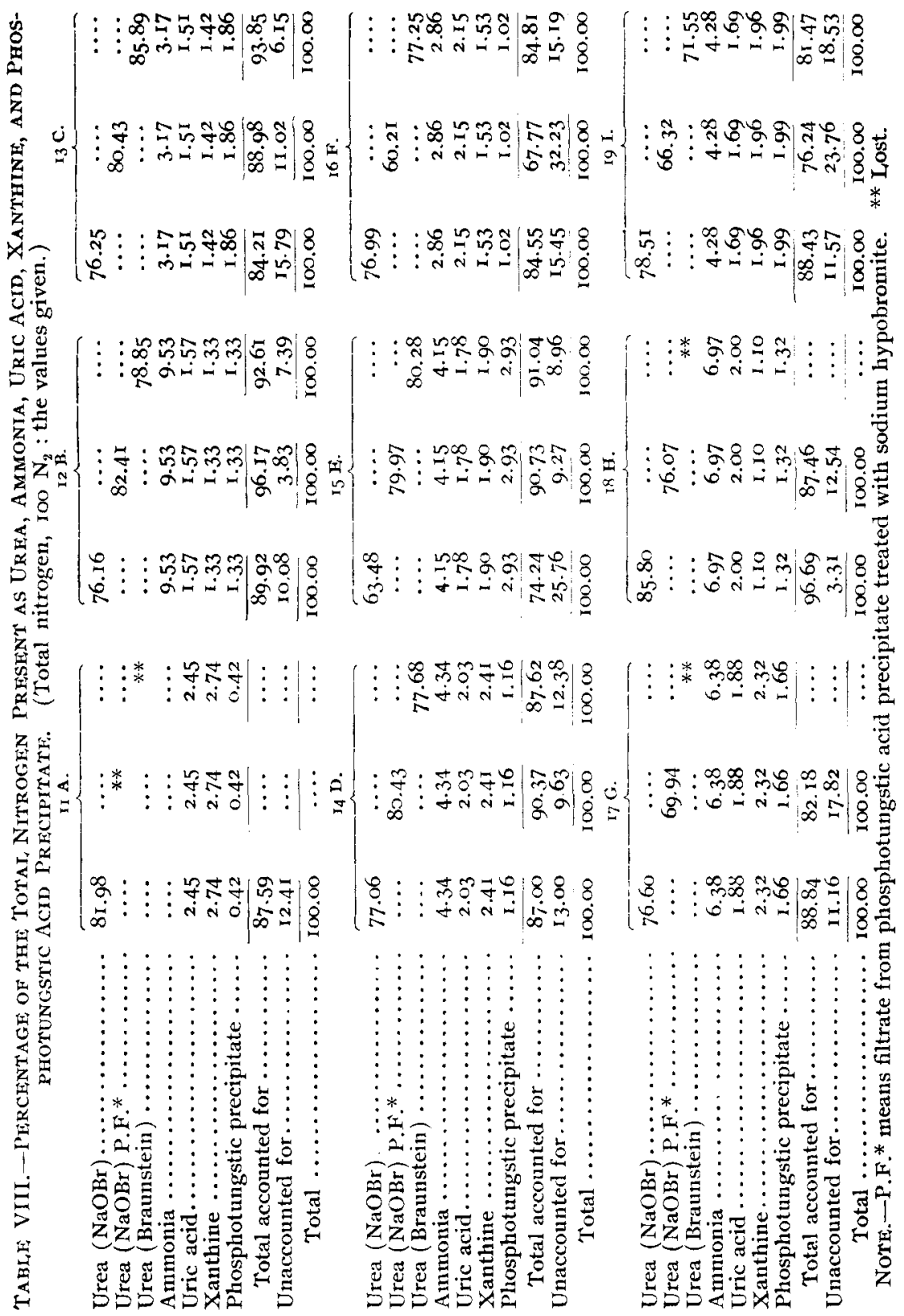


TABLE IX.-RATIOS.

\begin{tabular}{|c|c|c|c|c|}
\hline \multirow{3}{*}{$\begin{array}{c}\text { Date, } \\
\text { May, Igor. } \\
\text { II A }\end{array}$} & \multicolumn{3}{|c|}{$100 \mathrm{~N}_{2}:$} & \\
\hline & $\mathrm{P}_{2} \mathrm{O}_{5}$ & $\mathrm{SO}_{8}$ & cl. & \\
\hline & 20.0346 & I 8.6625 & 35.0809 & 107.3521 \\
\hline I2 B & 19.0092 & 17.1828 & 10.6831 & I 10.6293 \\
\hline $\mathrm{I}_{3} \mathrm{C}$ & 20.5429 & I 7.6695 & 19.7418 & II $6.26 \mathrm{I} 8$ \\
\hline I4 D & I 8.6210 & 17.5096 & II. 2060 & 106.3473 \\
\hline I5 E & 18.3565 & 17.4734 & 12.3607 & 105.0540 \\
\hline I6 F & 17.4114 & 17.6352 & 9.5298 & 98.7309 \\
\hline $17 \mathrm{G}$ & 18.0915 & 16.4067 & 22.9470 & โI0.2690 \\
\hline $18 \mathrm{H}$ & $19.145^{2}$ & I7.699I & $26.623^{\circ}$ & 108.1704 \\
\hline I9 I & 20.9494 & 17.8324 & 36.29 I 5 & I17.4793 \\
\hline$A, B, C$ & $19.872 \mathrm{I}$ & I 7.8388 & 23.9751 & I I I. 3984 \\
\hline $\mathrm{D}, \mathrm{E}, \mathrm{F}$ & 18.1547 & $I 7.5357$ & I1. 2368 & 103.5300 \\
\hline $\mathrm{G}, \mathrm{H}, \mathrm{I}$ & I 9.3064 & $17.25^{8} 7$ & 28.2207 & II 1.8644 \\
\hline
\end{tabular}

TABLE X.-THEOBROMINE AND PURINE BASES OBTAINED BY KRÜGER'S METHOD.

$\begin{array}{lcc}\text { Grams theobromine } & \begin{array}{c}\text { Grams copper } \\ \text { nitrogen. } \\ \text { precipitate, nitrogen. }\end{array} \\ \text { 13 C } & 0.0325 & 0.015 \mathrm{I} \\ \text { I4 D } & 0.0387 & 0.0027 \\ 15 \mathrm{E} & 0.1245 & 0.0001 \\ 16 \mathrm{~F} & 0.5742 & 0.0086 \\ 17 \mathrm{G} & 0.2932 & 0.004 \mathrm{I} \\ 18 \mathrm{H} & 0.0109 & 0.0064 \\ 19 \mathrm{I} & 0.0782 & 0.0106\end{array}$

In order to have a permanent record of a crystalline compound present in the urine during the period of overexertion, but not found in the urine of the other periods, except in the urine of the first day succeeding the middle period, microphotographs of the crystals were made, but their publication is withheld until the compound can be definitely identified. In appearance, these crystals closely resemble crystals of caffeine and caffeine nitrate; but the yield was too small to afford a quantity of pure crystals sufficient for a combustion. A nitrogen determination (by the absolute method) was made of a mixture of the crystals obtained from urines $D, E$, and $F$, by the Krïger ${ }^{1}$ method; but, on account of impurities present, the result is not conclusive beyond the demonstration of the presence of nitrogen in the compound.

In an attempt to observe the melting-point, the mass darkened but did not liquefy at $250^{\circ} \mathrm{C}$., but small needle-shaped crystals

I Martin Krïger: Loc. cit. 


\section{4}

were found in the upper part of the tube when examined under the microscope.

Upon incineration, the $D, E, F$ mixture yielded 63.43 per cent. ash (mostly sodium carbonate) and 36.57 per cent. (by difference) volatile organic substance.

0.0453 gram of substance gave $2.8 \mathrm{cc}$. nitrogen at $30^{\circ} \mathrm{C} ., 75 \mathrm{I}$ $\mathrm{mm}$., $=6.35$ per cent. nitrogen. On this basis, the organic matter volatilized contained 17.36 per cent. nitrogen. This is entirely too low for caffeine, but it closely approximates the amount of nitrogen found by Adolf Jolles ${ }^{1}$ in the phosphotungstic acid precipitate of theophylline (17.04 per cent. nitrogen). Jolles contends that phosphotungstic acid precipitates only that portion of the purine bases in which the nitrogen is attached to the methyl groups, and that the remainder of the base is split off as urea. Thus far we have macie no attempt to corroborate the work of Jolles, but shall give it attention in a subsequent investigation. On the basis of the original substance, the nitrogen found $(6.35$ per cent.) closely approximates the amount present in cynurenic acid- $\mathrm{C}_{10} \mathrm{H}_{7} \mathrm{NO}_{3}+\mathrm{H}_{2} \mathrm{O}=6.76$ per cent. nitrogen; but the large amount of ash precludes this substance, as does also the method by which the crystals were obtained.

The crystals obtained by the method of Harper-2 from measured quantities of the corresponding twenty-four hours urine were well defined (some of them could be plainly seen with the unaided eye) but a brownish "smear" soluble in chloroform contaminated them, and the quantity obtained was too small to provide an amount of pure crystals sufficient for a combustion.

The second method is so much simpler than that of Krüger that an attempt will be made shortly to secure large quantities of this crystalline compound with a view to definitely determine its constitution.

\section{SUMMARY.}

I. The influence of fatigue-stuffs upon the pulse and temperature is shown in Table I. Similar observations have been repeatedly made. The subject complained of headache during the afternoon of $D$ and the greater part of $E$ and $F$.

1 Adolf Jolles : Ber, d, chem. Ges., 33, 2 I I

$2 \mathrm{H}$. W. Harper: In the context of this communication-Analytical methods. 
2. Tables II, III, and IV are self-explanatory. The urine of the entire experiment exhibited an acid reaction, was normal in color and odor. As would be expected, the specific gravity curved upward during the middle period, and this period was also marked by a copious deposit of urates. The diazo reaction was practically uniform throughout the entire experiment. The reaction for indican was faint except in the $F$ and $G$ urines-these contained notable quantities; most marked in $\mathrm{G}$.

3. The income and outgo of nitrogen as shown in Table $V$ presents some very interesting features :

(a) In the food eaten the nitrogen content was largest in that of the first period, smallest in that of the middle period.

(b) The total nitrogen assimilated was largest in the third period, smallest in the middle period.

(c) The action of the digestive tract upon the nitrogenous food was greatest during the middle period, least during the first period. Of the total nitrogen in the food consumed during the successive periods 94 per cent. was appropriated during the first period, 97 per cent. during the second period, and 96 per cent. during the third.

(d) The output of nitrogen was largest during the middle period, smallest during the third period.

(e) In the nitrogen balance there was a decided loss during the middle period, a gain during the other two periods-the third period showing the largest gain. Taking the nine days' experiment as a whole the subject, made a small absolute gain in nitrogen. No permanent injury resulted from the experiment.

4. The results given in Tables VI and VIII are very interesting, but their discussion had best be postponed until an absolute method for the determination of urea, when mixed with its congeners, is devised.

5. While the results given in Tables VII and IX have no direct bearing upon the production of alkyl derivatives of xanthine during excessive muscular exercise they throw some light upon the chemical aspect of metabolism, and, therefore, are of value. The output of phosphoric anhydride was greatest during the first period of rest, and least during the last period. The subject did 
more mental work during the first period than during either of the subsequent periods. It should also be borne in mind that he suffered with headache during the middle period and the beginning of the third period. It is not claimed that the increase in the output of phosphoric anhydride during the first period is due to mental exertion. While such an idea naturally suggests itself, no precautions were taken to safeguard this point, and the data here presented are too meager to warrant a conclusion thereon.

The output of sulphuric anhydride was greatest during the middle period, and least during the last period. The reverse of this is shown in the case of the chlorine. The decrease in the chlorine output during the middle period is very marked, as is also its rise in the last period. As far as we know, this observation is entirely new. The amount of sodium chloride daily consumed was practically uniform throughout the three periods (nine days total). The osmotic movement from the blood to the cutaneous emunctories during the period of excessive exercise may, in part, explain the facts observed; but it should be remembered that the daily period of excessive muscular exertion was of short duration.

The table showing the relative amounts of $\mathrm{P}_{2} \mathrm{O}_{5}, \mathrm{SO}_{3}$ and $\mathrm{Cl}$ is particularly interesting. The IOO $\mathrm{N}_{2}: \mathrm{P}_{2} \mathrm{O}_{5}, \mathrm{Cl}$, and the IOO $\mathrm{SO}_{3}$ : $\mathrm{P}_{2} \mathrm{O}_{5}$, ratios seem to indicate that the role played by complexes containing these particular bodies is decidedly influenced by excessive exercise.

6. Evidence of the presence of methyl derivatives of xanthine is presented in Table $X$. This eyidence finds further support in the microphotographs of a crystalline substance obtained from the phosphotungstic acid precipitate by the method of Krüger, and of those obtained by the chloroform method. The quantity of crystals obtained in a state of purity was insufficient for a combustion. However, a nitrogen determination (by the absolute method) was made of some of the contaminated crystals that we might at least prove or disprove the presence of nitrogen in the compound. The results obtained are given. It will be seen that the nitrogen is too low for any of the known methyl derivatives of xanthine. In the context will be found a fuller discussion of this point. 
7. Thus far this investigation has thrown some new light upon the chemistry of fatigue, but much additional work is yet to be done before the theoretical discussion of the results may be profitably entered upon. The inquiry will be further pursued in this laboratory.

In conclusion the authors acknowledge their sincere thanks for assistance rendered by Messrs. F. C. Beall, O. D. Hargis, H. Kuehne, and M. B. Wesson.

NoTE.-This communication is dated June, I902, because the experimental part was complete by that time. However, the results were not put in their present shape for publication until September, 1902, the duties incident to the close of the session making it impossible to shape the results at an earlier date.

ThE UNIVERSTY OF TEXAS, Chemical LaboRatory, A Ustin, Texas, June, igoz.

\section{ON THE FIXATION OF AMMONIA AND POTASH BY HAWAIIAN SOILS.}

By J. T. CRAWLEY AND R. A. DUNCAN.

Received September 30, 1902.

In this Journal, 24, I I I4, will be found a paper by the writer on the "Fixation of Phosphoric Acid in the Soil." This paper will record results on the fixation of the other chief fertilizer constituents, ammonia and potash. The same methods described in that work were followed in these experiments, and the results, therefore, can be compared with each other. The main object is to find the depth in the soil at which the chief fertilizing materials are fixed and to determine the loss by drainage when the application is followed by a heavy irrigation. A given weight of the commercial article containing ammonia and potash, namely, sulphate of ammonia and sulphate of potash, is applied to the soil, and this is irrigated with 3 or 4 inches of water (the usual irrigation as practiced on the plantations), the drainage collected and analyzed. The difference between the amount applied and that found in the drainage is the amount abstracted or held by the soil. By varying the depth of soil we find the depth at which the ingredients are fixed. Boxes ( 9 inch cube) with holes in the bottom for the escape of water were used in the experiment. In all cases 